\title{
一种新型的共价有机骨架膜的制备与气体分离性能
}

\author{
付静茹贲腾* \\ (吉林大学化学学院 长春 130012)
}

\begin{abstract}
摘要 选用 2,5-二甲氧基对苯二甲酫和四胺基四苯甲烷利用溶剂热的方法在反应釜中制备得到一种新型的含有醚氧基 团的 3D 共价有机骨架(DMTA-COF). 通过理论模拟，粉末 X 射线衍射(PXRD)、傅里叶变换红外光谱(FT-IR)、热重分 析(TGA) 以及 $\mathrm{N}_{2}$ 吸附-脱附曲线等表征证实了其结构、热稳定性以及多孔性. 随后在聚苯胺(PANI)修饰的多孔氧化铝基 底表面原位生长了一层致密连续的 DMTA-COF 膜. 通过 XRD 和扫描电子显微镜(SEM)的表征证实了 DMTA-COF 膜具 有较高的结晶性以及良好的共生性. 由于极性的醚氧基团与电四极矩分子 $\mathrm{CO}_{2}$ 之间较强的相互作用进一步提高了希弗 碱类型的 DMTA-COF 对 $\mathrm{CO}_{2}$ 的亲和力, 从而增强了 DMTA-COF 膜的吸附扩散效应. 在常温常压下, DMTA-COF 膜的 $\mathrm{H}_{2} / \mathrm{CO}_{2}$ 双组分分离系数为 8.3 , 所对应的 $\mathrm{H}_{2}$ 渗透通量高达 $6.3 \times 10^{-7} \mathrm{~mol} \cdot \mathrm{m}^{-2} \cdot \mathrm{s}^{-1} \cdot \mathrm{Pa}^{-1}$.

关键词 共价有机骨架; 晶体结构; 膜; 气体分离; $\mathrm{H}_{2}$ 纯化
\end{abstract}

\section{Fabrication of a Novel Covalent Organic Framework Membrane and Its Gas Separation Performance}

\author{
Fu, Jingru Ben, Teng* \\ (College of Chemistry, Jilin University, Changchun 130012)
}

\begin{abstract}
Herein, we employ 2,5-dimethoxyterephthalaldehyde (DMTA) containing ether oxygen group in the structure as the construction unit to react with tetra-(4-anilyl)-methane (TAM) through Schiff-based condensation reaction in a Teflon-lined autoclave to synthesize a novel three-dimensional covalent organic framework named DMTA-COF. Furthermore, the condensation reaction was confirmed by Fourier transform infrared spectroscopy (FT-IR). The crystal structure of DMTA-COF was analyzed by the powder X-ray diffraction (PXRD) measurement in conjunction with structural simulation. The morphology, thermal stability, porosity and pore distribution of DMTA-COF were measured by scanning electron microscopy (SEM), thermogravimetric analysis (TGA), and $\mathrm{N}_{2}$ adsorption-desorption at $77 \mathrm{~K}$. The high affinity for $\mathrm{CO}_{2}$ adsorption was also confirmed by low pressure $\mathrm{CO}_{2}$ sorption. Considering the relatively small pore size and the strong $\mathrm{CO}_{2}$ adsorption interaction of DMTA-COF due to an abundant of ether oxygen group and imine linkage, we synthesized one continuous supported DMTA-COF membrane for $\mathrm{H}_{2} / \mathrm{CO}_{2}$ separation. In our study, the porous $\mathrm{Al}_{2} \mathrm{O}_{3}$ support surface was first coated with polyaniline (PANI) and was then further functionalized with aldehyde groups by reaction with DMTA at $150{ }^{\circ} \mathrm{C}$ for $1 \mathrm{~h}$. Finally, in situ growth of the COF membrane utilizing the covalent linkage yielded a novel continuous DMTA-COF membrane. X-ray diffraction (XRD) result indicated that the DMTA-COF membrane was pure phase and had high crystallinity. From SEM characterization, we could see that the DMTA-COF membrane was compact and well intergrowth and adhered to the support tightly. Gas separation performance results shown that DMTA-COF membrane had a high $\mathrm{H}_{2}$ permeance and selectivity of $\mathrm{H}_{2} / \mathrm{CO}_{2}$. For DMTA-COF membrane, the 1:1 binary mixture gas separation factors of $\mathrm{H}_{2} / \mathrm{CO}_{2}$ calculated as the gas molar ratios in permeate and retentate side was 8.3 at room temperature and atmospheric pressure. And $\mathrm{H}_{2} / \mathrm{CO}_{2}$ separation factor of DMTA-COF membrane exceeded the corresponding Knudsen coefficient (4.7), with $\mathrm{H}_{2}$ permeance of up to $6.3 \times 10^{-7} \mathrm{~mol} \cdot \mathrm{m}^{-2} \cdot \mathrm{s}^{-1} \cdot \mathrm{Pa}^{-1}$. Because of its outstanding characteristics, the novel DMTA-COF membrane is expected to be widely used in the field of $\mathrm{H}_{2}$ purification and separation.
\end{abstract}

Keywords covalent organic framework; crystal structure; membranes; gas separation; $\mathrm{H}_{2}$ purification

\section{1 引言}

与传统蒸馏相比，基于膜的气体分离技术具有操作 简便以及低能耗和高效率的优势 ${ }^{[1]}$. 分子篮分膜, 例如 分子篮 ${ }^{[2-4]}$ 、金属有机骨架 $(\mathrm{MOFs})^{[5-8]}$ 、固有微孔聚合物
$(\mathrm{PIMs})^{[9]}$ 或二维层状纳米材料均表现出了明显优于聚合 物膜的特有性质，例如同时具有高渗透通量和高选择性 的特点, 从而引起了人们广泛的关注和研究.

共价有机骨架(COFs) 是一类新型的结晶的多孔材 料, 它由纯有机的结构单元通过强的共价键构筑而

*E-mail: tben@jlu.edu.cn

Received April 28, 2020; published June 9, 2020.

Project supported by the National Natural Science Foundation of China (Nos. 91956108, 21871103), "111” Project (No. BP0719036) and the Science and Technology Department of Jilin Province Foundation (No. 20180414009GH).

项目受国家自然科学基金(Nos. 91956108, 21871103)、“111” 计划(No. BP0719036)和吉林省科技发展计划项目(No. 20180414009GH)资助. 
成 ${ }^{[10-15]}$. 有序的且可调控的孔结构, 大的比表面积和永 久的孔隙率, 功能的多样性以及超高的热稳定性和化学 稳定性以及特殊的吸附亲和力等独特的性质使得 COFs 有望成为具有高的气体分离选择性的膜材料 ${ }^{[16-18]}$. 尤其 是基于希弗碱类型连接 ${ }^{[19]}$ 的如亚胺连接的、连氮连接的 及腙连接 ${ }^{[20,21]}$ 的 COFs 表现出了比其他类型的 COFs 和 MOFs 更加优越的结构规律性以及化学稳定性 ${ }^{[22]}$. 因此 基于希弗碱类型连接的 COFs 也成为了制备具有高效的 气体分离膜的理想材料.

目前为止, COF 膜的制备方法主要包括共混法、原 位合成、层层堆叠和界面聚合等 ${ }^{[17]}$. 虽然利用共混法制 备得到的基于 $\mathrm{COF}$ 的混合基质膜(MMMs)在大大提高 气体渗透通量的基础上保留了聚合物的高的气体分离 选择性, 但是 COF 晶体和聚合物之间较差的兼容性容 易造成 COF 颗粒团聚和聚沉以及在 COF 晶体和聚合物 相接触的界面位置产生非选择性孔洞, 除此之外, COF 颗粒由于被聚合物完全包裹而容易导致孔道被阻塞，从 而不能完全发挥出 COF 的多孔性 ${ }^{[23]}$. 这些缺陷都限制 了基于 $\mathrm{COF}$ 的 MMMs 在气体分离领域的广泛应用. 利 用真空辅助的层层堆叠方法虽然操作简便, 且制备的 COF 膜的厚度可调控, 但是利用该方法制备 COF 膜的 前提是要通过超声剥离, 机械研磨等方法去剥离 $\mathrm{COF}$ 材料而制备得到 $\mathrm{COF}$ 纳米片. 在 $\mathrm{COF}$ 材料剥离的过程 中, 通常只能得到少量的纳米片, $\mathrm{COF}$ 纳米片的厚度很 难控制 ${ }^{[24]}$. 除此之外, 利用层层堆叠的方法制备的 COF 膜在干燥之后往往会出现裂痕, 严重影响了其应用. 而 且层层堆叠方法通常用于 2D COF 膜的制备, 这就使得 该方法具有很大的局限性. 人们虽然利用界面聚合的方 法在气一固 ${ }^{[25]}$ 、气一液 ${ }^{[26]}$ 以及水-有机 ${ }^{[27]}$ 界面处成功制备 得到了厚度可调控的超薄 COF 膜. 然而该方法制备得 到的 COF 膜机械性能较差, 在应用和表征之前需要把 COF 膜转移到相应的基底表面, 而转移过程中通常会 造成 COF 膜的碎裂和不平整, 从而在很大程度上影响 并限制了其应用.

与上述 COF 膜的制备方法相比，原位合成方法制 备得到的 COF 膜是连续的, 这就使得 $\mathrm{COF}$ 膜完全发挥 出了 COF 孔结构的所有潜在优势, 表现出了高的渗透 选择性 ${ }^{[28-30]}$. 此外, 支撑的 COF 膜具有较强的机械性 能, 可以在相对较高的压力条件下进行气体的分离, 大 大扩宽了气体分离膜的应用范围. 然而在基底上制备出 致密连续且具有高的分离选择性能的 COF 膜仍然面临 着巨大挑战. 其中一个原因是 $\mathrm{COF}$ 层和基底之间结合 力较差, 这就严重影响了无缺陷 $\mathrm{COF}$ 膜的制备. 另一个 原因是 $\mathrm{COF}$ 膜较大的孔尺寸. 膜分离大多数都是基于 尺寸篮分的, 因此 COFs 的孔尺寸影响甚至决定着 COF 膜的平均孔尺寸, 进而决定着 COF 膜的应用. 目前, 已 报道的基于希弗碱类型的多孔 COFs 的孔尺寸 $(0.8 \sim 5$ $\mathrm{nm})^{[15,31]}$, 这比气体分子的动力学直径 $(0.25 \sim 0.5 \mathrm{~nm})$ 大
得多，所以 COF 膜材料的孔尺寸严格限制了 COF 膜的 制备以及在气体分离领域的应用. 目前增强 COF 膜的 气体分离选择性的方法主要有调控 COF 膜的孔尺寸, 增强尺寸篮分效应，例如 Caro 等 ${ }^{[29]}$ 制备了 COF-COF 复 合膜，形成了相互交错的孔道，有效地降低了 COF 膜的 有效孔尺寸，从而增强了 $\mathrm{COF}$ 膜的气体渗透分离选择 性; 另外一种方法是有目的地选择具有选择吸附作用的 膜材料，增强吸附效应，例如 Caro 和 Fan 等 ${ }^{[30]}$ 选取聚醚 酰亚胺(PEI)修饰多孔氧化铝基底制备了 ACOF-1 膜, 最 终基于 ACOF-1 对 $\mathrm{CO}_{2}$ 的选择性吸附作用和尺寸篎分效 应的协同作用, 增强了 $\mathrm{CO}_{2} / \mathrm{CH}_{4}$ 的分离性选择性. 因此, 具有气体选择性吸附作用且孔尺寸较小的 COF 膜材料 的制备是增强气体分离选择性的关键.

选取 2,5-二甲氧基对苯二甲醛(DMTA)和四胺基四 苯甲烷(TAM)通过溶剂热的方法制备得到了一种新型的 含有醚氧基团的希弗碱类型的 3D COF, 如图式 1 所示. DMTA-COF 具有较小的孔尺寸 (1.06 nm), 由于骨架结 构中含有大量的醚氧基团以及亚胺键, 表现出高的 $\mathrm{CO}_{2}$ 选择性吸附作用. 基于此, 以聚苯胺修饰多孔氧化铝基 底，利用共价功能化连接，在基底表面制备了一层致密 连续的 DMTA-COF 膜, 气体分离测试结果表明, DMTA-COF 膜具有良好的 $\mathrm{H}_{2}$ 渗透选择性.<smiles>COc1cc(C=O)c(OC)cc1C=O</smiles>

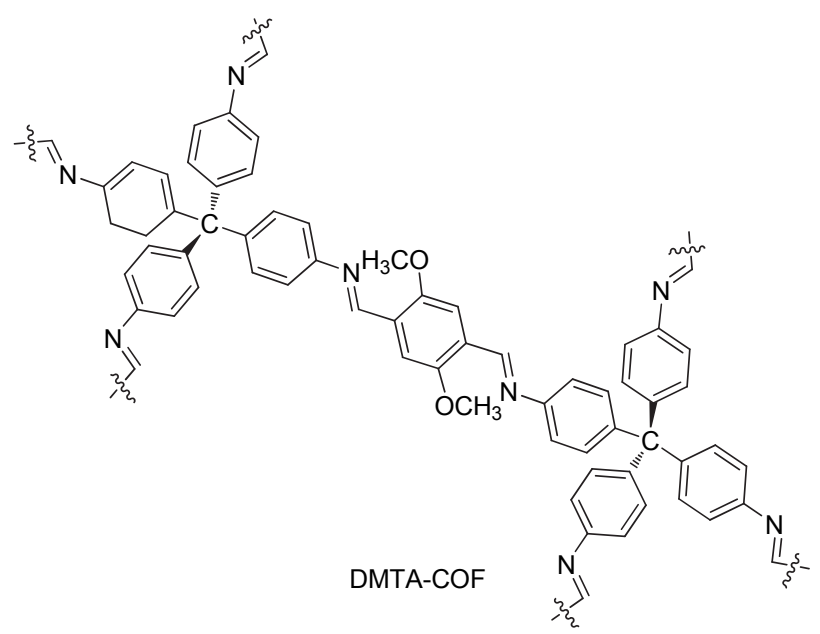

图式 1 DMTA-COF 的合成

Scheme 1 Synthesis of DMTA-COF 


\section{2 结果与讨论}

\subsection{DMTA-COF 粉末的表征}

\subsubsection{X 射线衍射分析}

为了表征 DMTA-COF 的结晶性, 对在反应釜中制 备的 DMTA-COF 粉末进行了 XRD 测试, 如图 1 和图 2a 所示. 从图 1 中可以看出产物 DMTA-COF 的 XRD 图与 单体(DMTA, TAM)相比发生了明显的变化, 具有微孔 性质，说明 2,5-二甲氧基对苯二甲醛和四胺四苯甲烷两 种单体确实是发生了聚合反应, 得到了结晶的微孔聚合 物. 另外, DMTA-COF 的衍射峰尖锐, 强度较高, 说明 COF 具有很高的结晶性, 晶体的规整度较高. 此外脱去 溶剂活化处理后的 DMTA-COF 仍然具有较高的结晶性 (图 2a), 说明骨架脱去溶剂后仍然可以保持, 没有塌陷, 也表明 DMTA-COF 具有较高的稳定性.

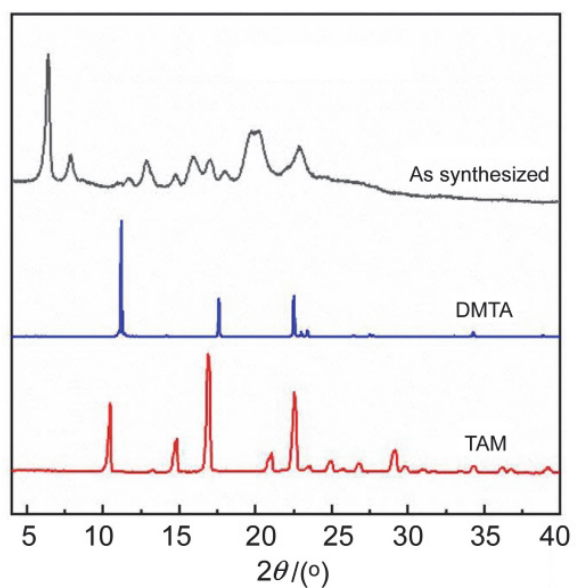

图 1 新合成的 DMTA-COF, 2,5-二甲氧基对苯二甲醛(DMTA)以及四 胺基四苯甲烷(TAM)的粉末 XRD 图

Figure 1 PXRD patterns of as-synthesized DMTA-COF powder, 2,5-dimethoxyterephthalaldehyde (DMTA) and tetra-(4-anilyl)-methane (TAM)

通过粉末 X 射线衍射测试对三维 DMTA-COF 的晶 体结构进行了确定, 并且结合 Materials Studio 7.0 软件 包对 COF 结构进行模拟(图 2). 基于三重穿插 dia 拓扑, 对 DMTA-COF 的结构模型进行了几何能量优化后, 得 到了 DMTA-COF 的晶胞参数(空间群: I41/A, $a=b=$ $2.838 \mathrm{~nm}, c=1.701 \mathrm{~nm}, \alpha=\beta=\gamma=90^{\circ}$ ). 除此之外, 还对 实验测得的 XRD 数据进行了 Pawley 精修, 结果表明精 修结果与实验测得的 XRD 除了具有细微的差别, 大多 数主要的衍射峰都能够很好地匹配 $\left(R_{\mathrm{wp}}=5.13 \%, R_{\mathrm{p}}=\right.$ $2.91 \%$ )(图 2). 这主要是由于 COFs 骨架具有呼吸效应 ${ }^{[32]}$ 其实际实验测得的 XRD 并不仅仅是由晶胞结构所确定 的还和其是否含有溶剂分子以及所含有的溶剂分子种 类有关. 而 DMTA-COF 的实验所测得的 XRD 是在抽真 空脱去溶剂分子后测得的, 其在抽真空脱去溶剂分子的 过程中可能会导致骨架的某些精细结构发生变化, 从而 导致实验测得的 XRD 会和晶胞模拟的 XRD 有些细微偏
差.

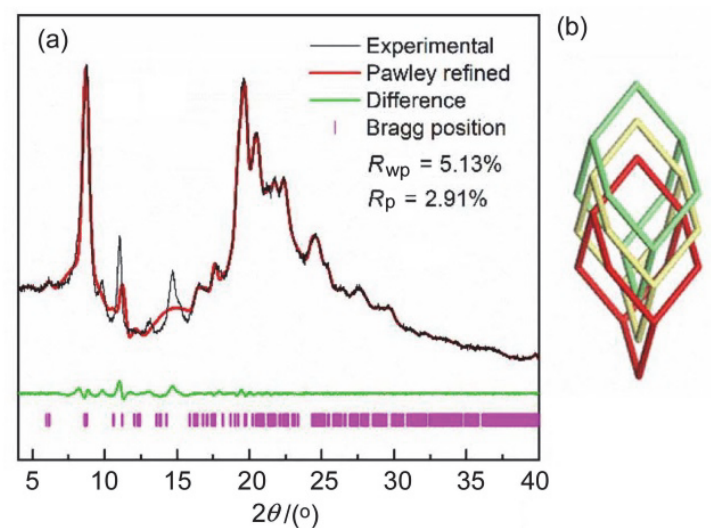

图 2 (a)活化后 DMTA-COF 的 XRD 图; (b) DMTA-COF 的三重穿插 dia 拓扑结构

Figure 2 (a) XRD patterns of activated DMTA-COF; (b) 3-fold interpenetrated dia net of DMTA-COF

\subsection{2 傅里叶变换红外光谱和热重分析}

为了进一步确定 DMTA-COF 的结构组成, 我们对 其进行了傅里叶变换红外光谱的表征. 如图 3a 所示, DMTA-COF 对比于单体在 $1620 \mathrm{~cm}^{-1}$ 出现了新的红外特 征吸收峰，该峰对应于 $\mathrm{C}=\mathrm{N}$ 的伸缩振动，说明单体之 间确实是发生了醛胺缩合反应, 形成了亚胺类的 COF. 除此之外, DMTA-COF 在 $1659 \mathrm{~cm}^{-1}$ 出现了对应于羰基 的红外特征峰, 强度很弱, 说明 DMTA-COF 端基为醛 基. 接着我们对活化好的 DMTA-COF 在空气氛围下进 行了热重分析(图 3b). 从图中可以看出经过无水四氢呋 喃溶剂交换以及在 $120{ }^{\circ} \mathrm{C}$ 抽真空干燥 $12 \mathrm{~h}$ 后的样品, 没有溶剂失重过程，表明活化处理后的 DMTA-COF 完 全脱去了溶剂. 此外, 在 $413{ }^{\circ} \mathrm{C}$ 之前, 材料没有超过 $5 \%$ 的失重, 表明 DMTA-COF 具有较高的热稳定性.

\subsection{3 气体吸附测试}

为了表征 DMTA-COF 的多孔性, 我们利用氮气吸 附-脱附实验对 3D DMTA-COF 材料的孔分布以及比表 面积进行了测试. $77 \mathrm{~K}$ 下氮气吸附测试结果表明 DMTA-COF 在较低的相对压强 $\left(p / p_{0}<0.05\right)$ 范围内气体 吸附量急剧上升, 说明 DMTA-COF 是典型的微孔材料. 吸附曲线和脱附曲线没有完全闭合, 并且表现出了明显 的迟滞现象，这在以往报道的关于 $\mathrm{COF}$ 的文献中也有 类似结果 ${ }^{[33]}$, 这主要归因于 $\mathrm{N}_{2}$ 吸附过程中在压力增加 下由客体分子诱导的 $\mathrm{COF}$ 结构转变和客体分子在 $\mathrm{COF}$ 骨架中的重新定向堆积 ${ }^{[33-35]}$. 通过计算得到 DMTACOF 的 BET 比表面积为 $374 \mathrm{~m}^{2} \cdot \mathrm{g}^{-1}$, 应用非定域密度泛 函理论(NLDFT)计算得到 DMTA-COF 的孔分布主要集 中在 $1.06 \mathrm{~nm}$ (图 4).

为了探究 DMTA-COF 的气体存储和选择性吸附能 力, 我们在不同的温度下对其进行了 $\mathrm{H}_{2}, \mathrm{CO}_{2}, \mathrm{CH}_{4}$ 的吸 附脱附曲线的测试并计算了对不同气体的吸附焓(图 5). 

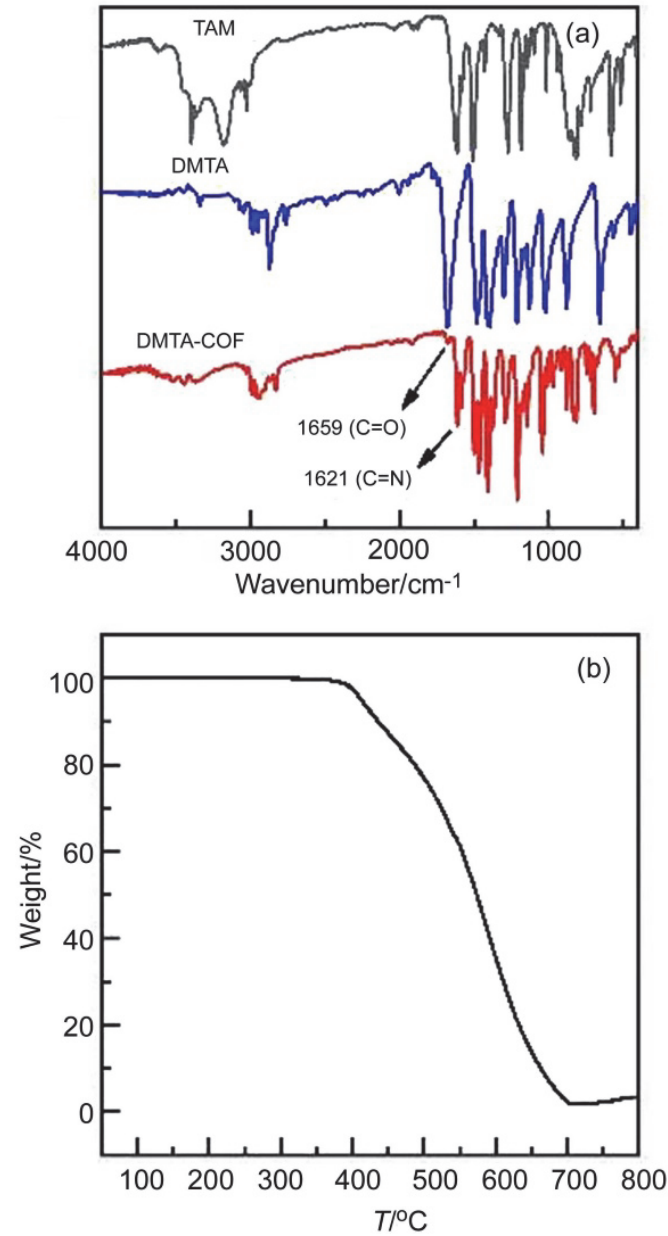

图 3 (a) TAM, DMTA 以及 DMTA-COF 的 FT-IR 图; (b)在空气氛围 下 DMTA-COF 的热重分析图

Figure 3 (a) FT-IR patterns of TAM, DMTA and DMTA-COF; (b) TGA analysis pattern of DMTA-COF in air atmosphere

从图 $5 \mathrm{a} \sim 5 \mathrm{c}$ 可以看出, DMTA- $\mathrm{COF}$ 对 $\mathrm{H}_{2}, \mathrm{CO}_{2}, \mathrm{CH}_{4}$ 具有 一定的吸附量, 表现出了一定的气体存储能力. 在 100 $\mathrm{kPa}$ 下, DMTA-COF 在 77 和 $87 \mathrm{~K}$ 下对 $\mathrm{H}_{2}$ 的吸附量分别 为 $63.05,30.62 \mathrm{~cm}^{3} \cdot \mathrm{g}^{-1}$; 在 273 和 $298 \mathrm{~K}$ 下对 $\mathrm{CO}_{2}$ 的吸 附量分别为 $16.11,10.27 \mathrm{~cm}^{3} \cdot \mathrm{g}^{-1}$; 在 $273 \mathrm{~K}$ 和 $298 \mathrm{~K}$ 下 对 $\mathrm{CH}_{4}$ 的吸附量分别为 $6.58,4.68 \mathrm{~cm}^{3} \cdot \mathrm{g}^{-1}$. 为了评估 DMTA-COF 对不同气体的吸附能力的大小, 通过测试 两个不同温度下的吸附量, 我们利用仪器自带软件计算 得到了 DMTA-COF 对不同气体的吸附焓. 从图 $5 \mathrm{~d} \sim 5 \mathrm{f}$ 可以看出, DMTA-COF 对 $\mathrm{H}_{2}, \mathrm{CO}_{2}, \mathrm{CH}_{4}$ 的吸附焓分别为 $8.0,23.0,10.5 \mathrm{~kJ} \cdot \mathrm{mol}^{-1}$. 吸附焓可以用来评估一种材料 对气体的吸附能力, 吸附焓越大, 则材料对气体的吸附 作用力越强. 因此, 从 DMTA-COF 对不同气体的吸附 焓可以看出, DMTA-COF 对 $\mathrm{CO}_{2}$ 的吸附能力最强, 这是 由于其骨架中含有大量的醚氧基团以及亚胺键, 对 $\mathrm{CO}_{2}$ 具有强的选择性吸附作用 ${ }^{[36,37]}$.
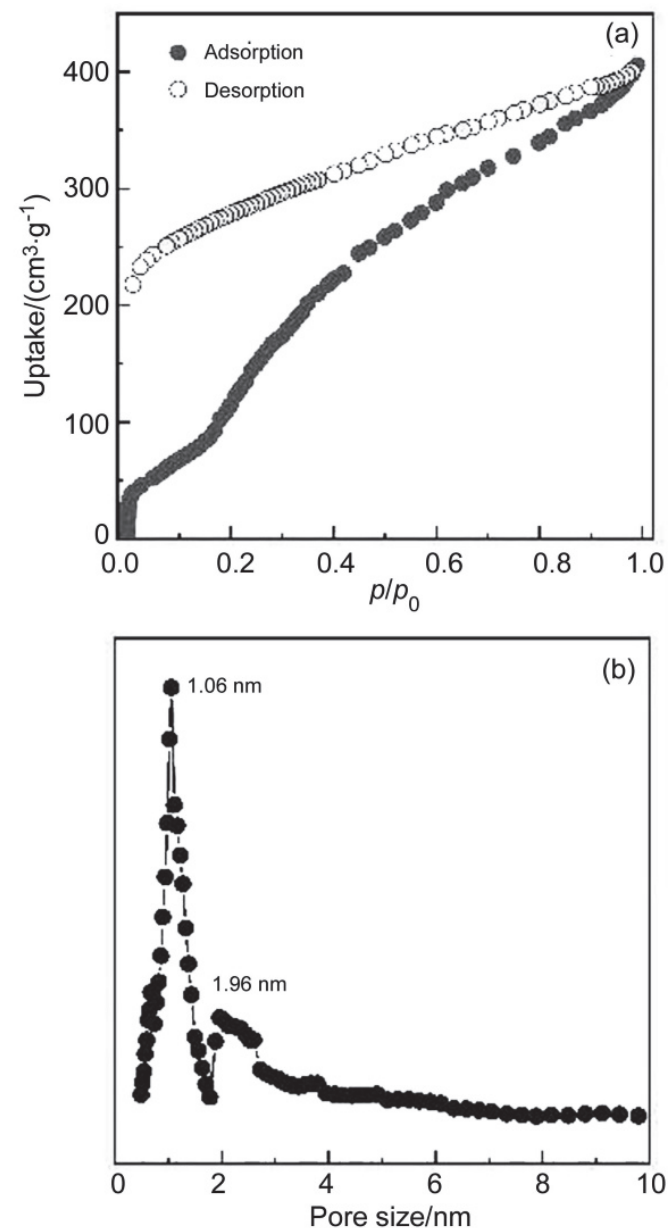

图 4 (a) $77 \mathrm{~K}$ 下 DMTA-COF 的 $\mathrm{N}_{2}$ 吸附-脱附曲线; (b) DMTA-COF 的孔径分布

Figure 4 (a) $\mathrm{N}_{2}$ sorption-desorption isotherm of DMTA-COF at $77 \mathrm{~K}$; (b) the pore size distribution for DMTA-COF

\subsubsection{SEM 测试}

从 DMTA-COF 的 XRD 以及气体吸附测试结果可 知, DMTA-COF 具有很高的结晶性. 因此, 进一步通过 SEM 对其形貌、晶体尺寸大小进行了测试. 从图 6 的 SEM 图可以看出 DMTA-COF 晶体为 “大米” 状, 形貌 较为均一, 晶体尺寸约为 $6 \mu \mathrm{m}$.

\subsection{DMTA-COF 膜的表征}

DMTA-COF 膜的制备流程图如图 7所示, 本章中选 取多孔氧化铝为基底, 首先利用聚苯胺对其表面进行修 饰，随后通过聚苯胺和 2,5-二甲氧基对苯二甲醛中的醛

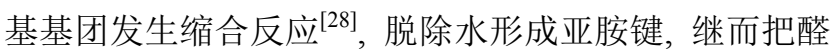
单体吸附和嫁接在基底的表面，完成对基底表面的醛基 化处理，为接下来 DMTA-COF 在基底表面的生长提供 异相成核位点. 随后基底表面自由的醛基与溶液中的四 胺基四苯甲烷进一步发生醛胺缩合反应，从而参与到整 个的 DMTA-COF 晶体的生长过程中, 最终在基底表面 生长了一层致密连续的 DMTA-COF 膜(图式 2). 

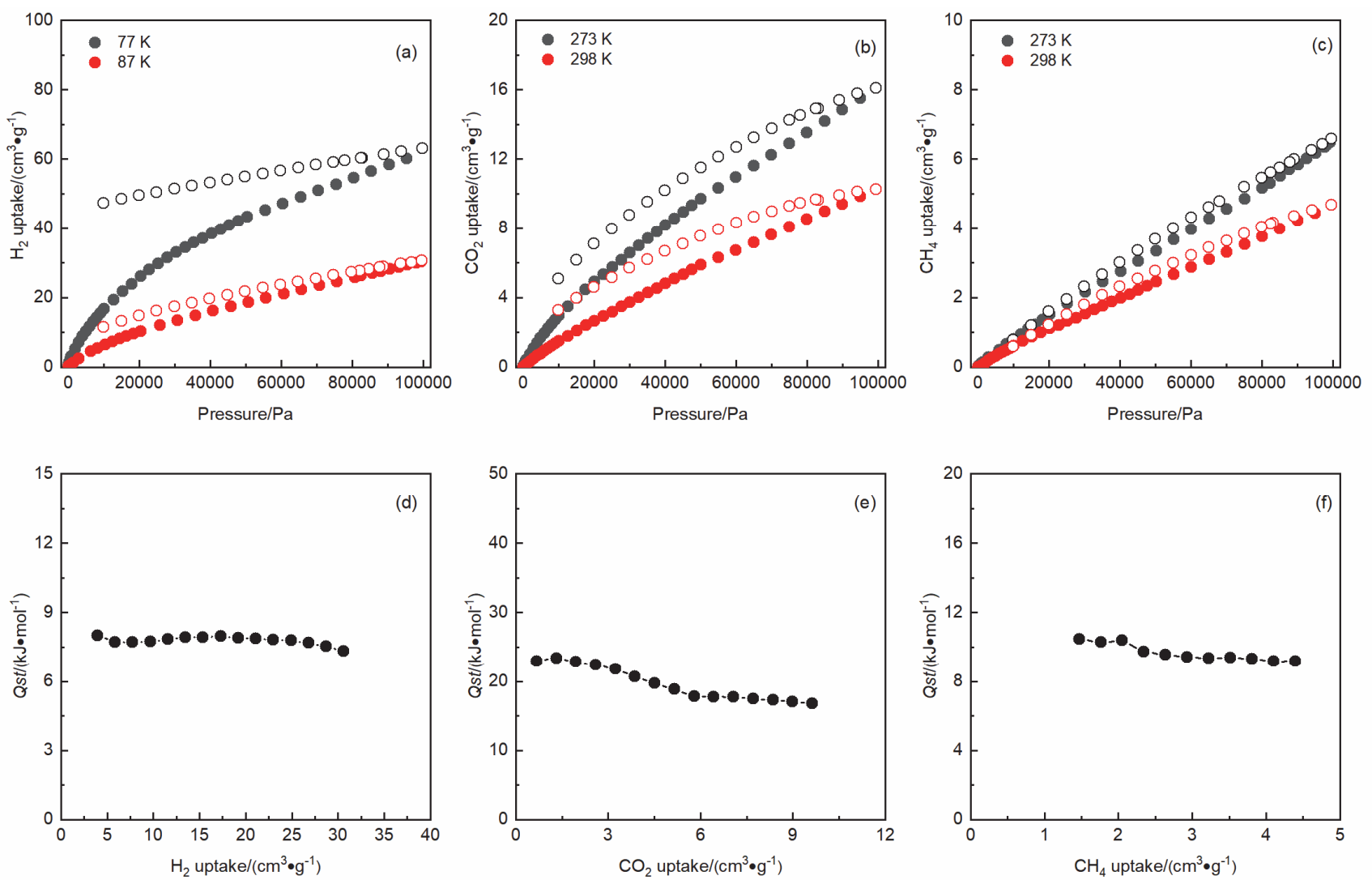

图 5 脱气后的 DMTA-COF 粉末的 $\mathrm{H}_{2}, \mathrm{CO}_{2}, \mathrm{CH}_{4}$ 的吸附等温线(实心: 吸附, 空心: 脱附)( $\mathrm{a}, \mathrm{b}, \mathrm{c}$ )和 $\mathrm{H}_{2}, \mathrm{CO}_{2}, \mathrm{CH}_{4}$ 的吸附焓(d,e,f)

Figure $5 \mathrm{H}_{2}, \mathrm{CO}_{2}$ and $\mathrm{CH}_{4}$ sorption isotherms (solid symbols: adsorption, open symbols: desorption) (a, b, c) and isoteric enthalpy $Q_{\text {st }}$ of $\mathrm{H}_{2}, \mathrm{CO}_{2}$ and $\mathrm{CH}_{4}$ adsorption (d, e, f) respectively for degassed DMTA-COF powder
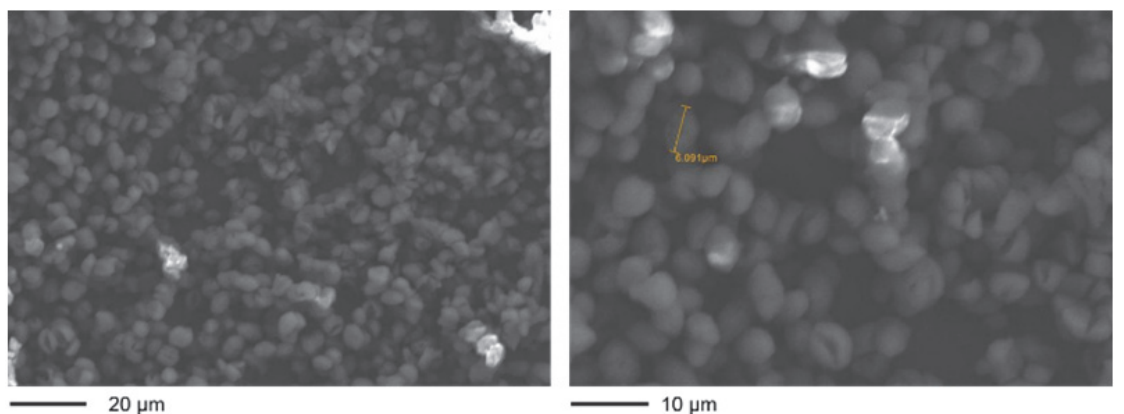

图 6 DMTA-COF 的 SEM 图

Figure 6 SEM pictures of DMTA-COF

\subsubsection{DMTA-COF 膜的结晶性表征}

为了表征 DMTA-COF 膜的结晶性, 我们对新制备 没有经过去溶剂活化处理的 DMTA-COF 膜进行了 XRD 的表征. 从图 8 可以看出, 除了来自多孔 $\mathrm{Al}_{2} \mathrm{O}_{3}$ 基底以 及 $\mathrm{Al}$ 质模具的 XRD 特征峰之外, DMTA-COF 膜的 XRD 图与 DMTA-COF 粉末的 XRD 完全相匹配, 没有多余的 杂峰, 并且峰强度较大, 说明我们在氧化铝基底上成功 制备了 DMTA-COF 膜, 该膜是纯相的, 并且具有高度 的结晶性.

\subsubsection{DMTA-COF 膜的 SEM 表征}

为了表征 DMTA-COF 膜的形貌, 我们首先对其进 行了直观上的表征(图 9a). 从 DMTA-COF 膜的光学照 片可以看出, 黄色的 COF 层把基底上的蓝色的 PANI 层 完全覆盖, 并且膜的表面没有肉眼可见的裂痕或孔洞等 缺陷, 说明从直观上看 DMTA-COF 膜是致密连续的.

图 $9 b$ 为基底的正面图, 从中可以看出即使在高倍 数下其表面仍然较为平整, 并且具有多孔性质. 光滑平 整的基底为 DMTA-COF 膜在其表面的生长提供了保障, 而多孔性则避免了在气体分离过程中基底对气体渗透 的阻碍. 为了进一步表征 DMTA-COF 膜微观致密连续 


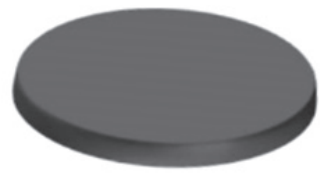

Porous $\mathrm{Al}_{2} \mathrm{O}_{3}$ support

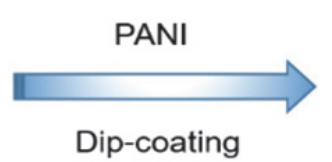

Dip-coating

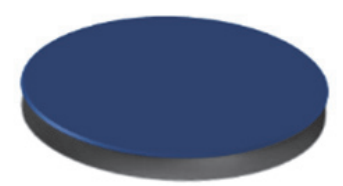

PANI-modified porous $\mathrm{Al}_{2} \mathrm{O}_{3}$ support
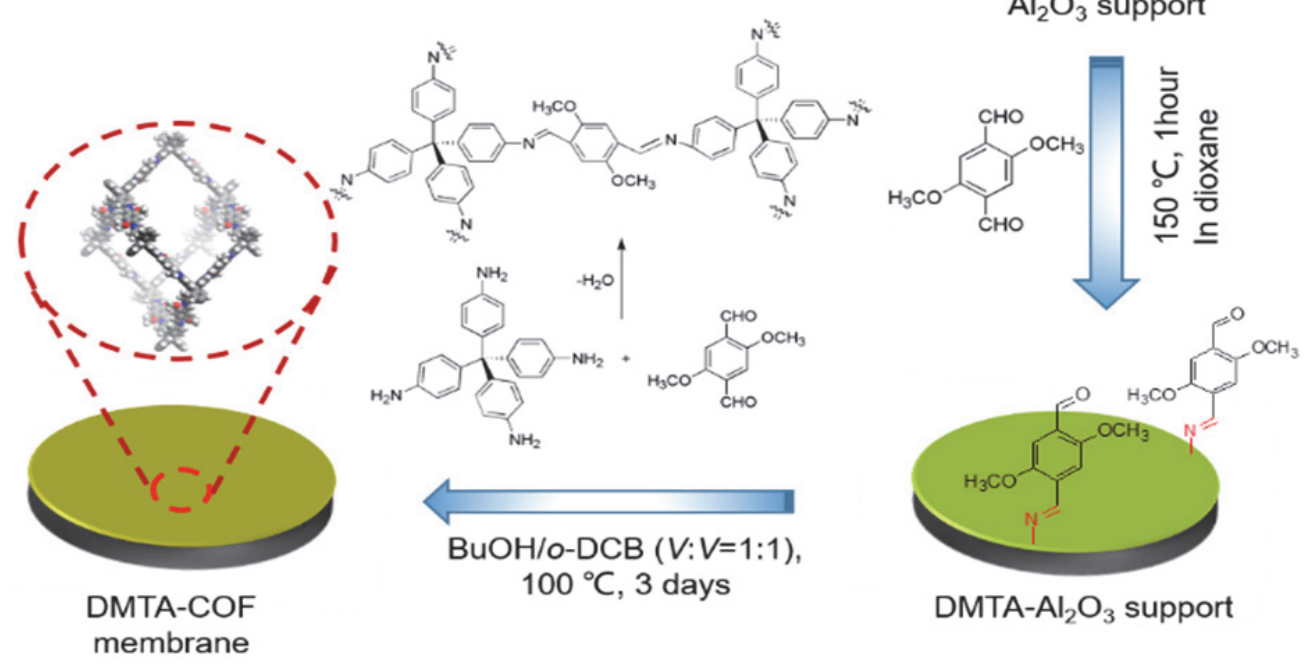

图 7 DMTA-COF 膜的制备流程图

Figure 7 Schematic representation of synthesis of DMTA-COF membrane<smiles>CC[N+](=Cc1cc(OC)c(C=O)cc1OC)c1cc(OC)c(C=O)cc1OC</smiles><smiles>Nc1ccc(C(c2ccc(N)cc2)(c2ccc(N)cc2)c2ccc(N)cc2)cc1</smiles><smiles>COc1cc(/C=N/[N+](C)([O-])c2ccc(C)cc2)c(OC)cc1C=O</smiles><smiles></smiles>

图式 2 DMTA-COF 膜合成过程中发生的化学反应

Scheme 2 The chemical reactions that take place during the fabrication of the DMTA-COF membrane

性和厚度, 对其正面与截面分别进行了 SEM 分析(图 9c, 9d). 从正面图中, 可以看出 DMTA-COF 晶体并不是单 独散落在基底上, 而是晶体与晶体之间共生性良好, 没 有明显的裂痕以及针孔等缺陷, 说明 DMTA- COF 膜具 有较好的连续性. 从其截面图同样可以看出晶体之间具 有较好的共生性, 膜厚度约为 $20 \mu \mathrm{m}$. 此外 DMTA-COF 膜和基底之间没有裂缝出现, 而是紧密结合在一起, 说 明 DMTA-COF 膜与基底之间具有强的结合力. 这是由 于 PANI 修饰基底为之后的 2,5-二甲氧基对苯二甲醛在 基底表面的嫁接提供了保障, 而嫁接在基底上的这一部
分醛单体继续与反应液中的四胺四苯甲烷反应, 为 DMTA-COF 膜在基底上的生长提供了成核位点, 不仅 确保了 DMTA-COF 膜的连续性, 也增强了 DMTA-COF 与基底之间的作用力, 最终形成了高质量的 DMTA-COF 膜.

\subsubsection{DMTA-COF 膜气体分离性能分析}

对完全脱溶剂后的 DMTA-COF 膜进行了单组分渗 透和双组分气体分离的测试. 单组分气体 $\mathrm{H}_{2}, \mathrm{CO}_{2}, \mathrm{~N}_{2}$, $\mathrm{CH}_{4}$ 以及等物质的量的双组分气体 $\mathrm{H}_{2} / \mathrm{CO}_{2}, \mathrm{H}_{2} / \mathrm{N}_{2}, \mathrm{H}_{2}$ / $\mathrm{CH}_{4}$ 的体积流速均利用 Wicke-Kallenbach 技术进行测试 


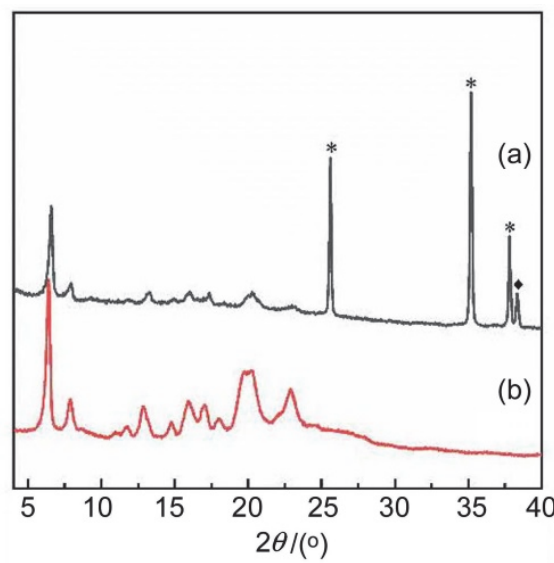

图 8 新合成的 DMTA-COF 膜(a)和 DMTA-COF 粉末(b)的 XRD 图(*: $\mathrm{Al}_{2} \mathrm{O}_{3}$ 基底的信号; $: \mathrm{Al}$ 模具的信号

Figure 8 XRD patterns of as synthesized DMTA-COF membrane (a) and DMTA-COF powder (b) (*: $\mathrm{Al}_{2} \mathrm{O}_{3}$ support signal; $\bullet$ : Al mold signal)
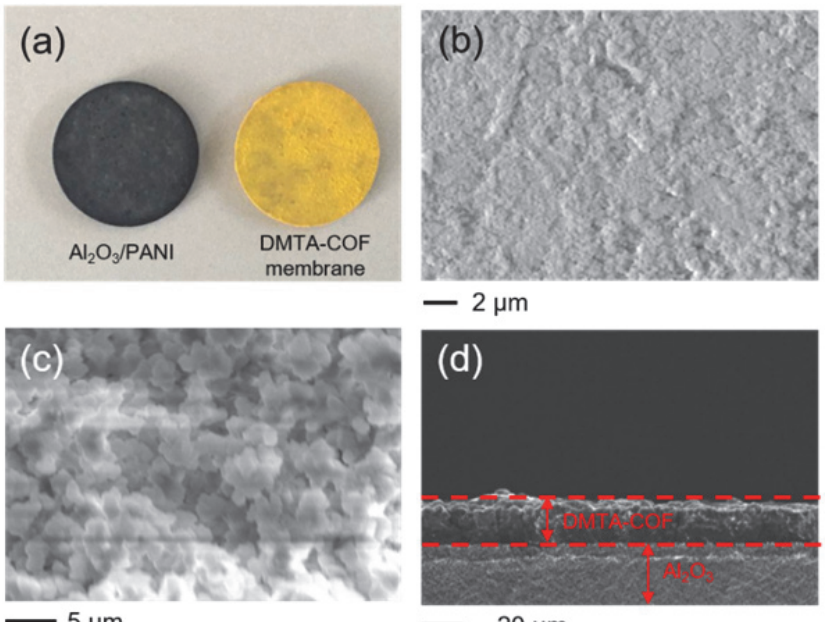

$-5 \mu \mathrm{m}$

- $20 \mu \mathrm{m}$

图 $9 \mathrm{Al}_{2} \mathrm{O}_{3} / \mathrm{PANI}$ 和 DMTA-COF 膜的光学照片(a); $\mathrm{Al}_{2} \mathrm{O}_{3}$ 基底的正面 SEM 图(b); DMTA-COF 膜的正面(c)和截面(d)SEM 图

Figure 9 The digital photographs of $\mathrm{Al}_{2} \mathrm{O}_{3} / \mathrm{PANI}$ and DMTA-COF membrane (a); SEM picture of the top view of $\mathrm{Al}_{2} \mathrm{O}_{3}$ support (b); SEM pictures of the top view (c) and cross-sectional view (d) of DMTA-COF membrane

(图 10). 图 11 是在常温常压下, DMTA-COF 膜的单组分 气体 $\left(\mathrm{H}_{2}, \mathrm{CO}_{2}, \mathrm{~N}_{2}, \mathrm{CH}_{4}\right)$ 的渗透通量与所对应的气体动力 学直径的关系图. $\mathrm{H}_{2}, \mathrm{CO}_{2}, \mathrm{~N}_{2}, \mathrm{CH}_{4}$ 的动力学直径分别为 $0.29,0.33,0.36$ 和 $0.38 \mathrm{~nm}$. 如图 11 所示, 四种气体穿透 DMTA-COF 膜的通量大小顺序为 $\mathrm{H}_{2}>\mathrm{CH}_{4}>\mathrm{N}_{2}>\mathrm{CO}_{2}$. $\mathrm{H}_{2}, \mathrm{CO}_{2}, \mathrm{~N}_{2}, \mathrm{CH}_{4}$ 的渗透通量依次为 $6.6 \times 10^{-7}, 6.8 \times$ $10^{-8}, 1.3 \times 10^{-7}, 2.1 \times 10^{-7} \mathrm{~mol} \cdot \mathrm{m}^{-2} \cdot \mathrm{s}^{-1} \cdot \mathrm{Pa}^{-1}$ (表 1 ). $\mathrm{H}_{2}$ 的 渗透通量最高, 这可归因于 $\mathrm{H}_{2}$ 的动力学直径最小, 较其 他气体更容易更快地穿透膜材料; 另一原因是 DMTA$\mathrm{COF}$ 对 $\mathrm{H}_{2}$ 的吸附作用较弱 $\left(Q s t=8.0 \mathrm{~kJ} \cdot \mathrm{mol}^{-1}\right)$ (图 5), 所 以 $\mathrm{H}_{2}$ 在膜材料中扩散时, 其渗透不会由于 $\mathrm{H}_{2}$ 被膜材料 骨架吸附而阻碍. 值得说明的是, $\mathrm{CO}_{2}$ 的动力学直径比

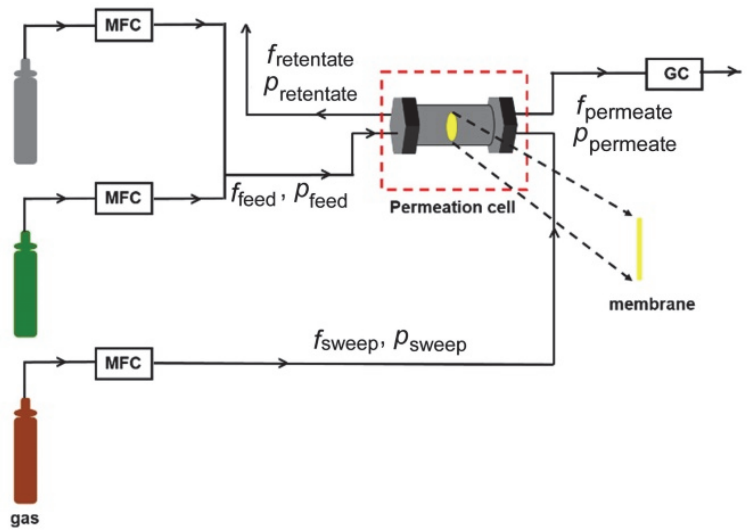

图 10 单组分和混合气体渗透测试装置

Figure 10 Measurement equipment for both single and mixed gas permeation (MFC: Mass flow controller; GC: Gas chromatograph; $f$ : volumetric flow rate; $p$ : pressure)

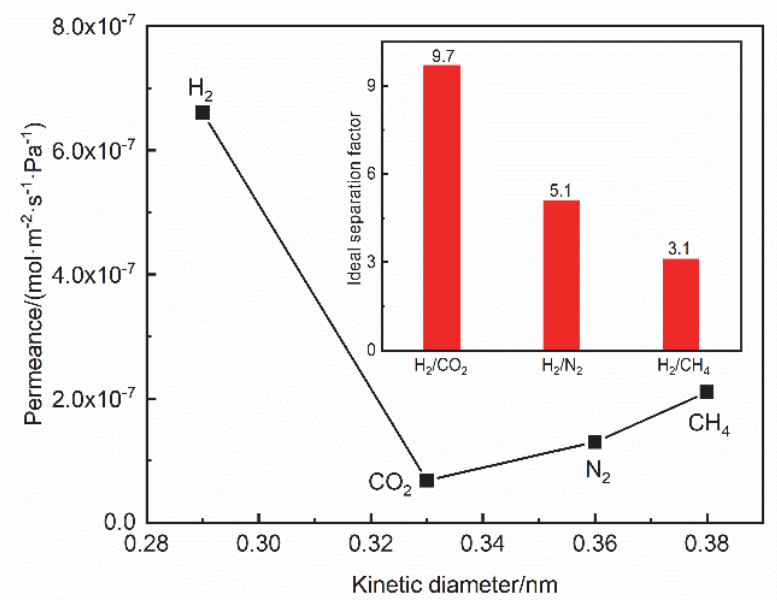

图 11 常温常压下, 不同的单组分气体穿透 DMTA-COF 膜的渗透通 量与所对应的气体动力学直径的关系. 插图为 DMTA-COF 膜的理想 分离系数

Figure 11 Single gas permeance of various gases through the DMTACOF membrane at room temperature and atmospheric pressure as a function of their kinetic diameter. The inset shows the ideal separation factors of the DMTA-COF membranes

$\mathrm{N}_{2}$ 和 $\mathrm{CH}_{4}$ 的小, 却有着最小的渗透通量. 这是由于 DMTA-COF 骨架结构中含有大量的醚氧基团以及亚胺 键，而极性的醚氧基团和亚胺键与 $\mathrm{CO}_{2}$ 之间具有强的电 四极矩相互作用 ${ }^{[36,37]}$, 这就导致 DMTA-COF 具有高的 $\mathrm{CO}_{2}$ 亲和力和捕获能力. 从图 5 也可以看出, DMTA$\mathrm{COF}$ 对 $\mathrm{CO}_{2}$ 的吸附焓 $\left(23.0 \mathrm{~kJ} \cdot \mathrm{mol}^{-1}\right)$ 远大于 $\mathrm{CH}_{4}$ 的吸附 焓 $\left(10.5 \mathrm{~kJ} \cdot \mathrm{mol}^{-1}\right)$, 所以在 $\mathrm{CO}_{2}$ 穿透 DMTA-COF 膜的过 程中, 由于 $\mathrm{CO}_{2}$ 与 DMTA-COF 膜具有较强的吸附相互 作用, 大量的 $\mathrm{CO}_{2}$ 分子被吸附在 DMTA-COF 孔道中, 阻碍了 $\mathrm{CO}_{2}$ 的顺利通过, 从而导致了 $\mathrm{CO}_{2}$ 的渗透通量较 低. 膜材料的理想分离系数由两种气体的渗透通量之比 求得，通过计算，DMTA-COF 膜的 $\mathrm{H}_{2} / \mathrm{CO}_{2}, \mathrm{H}_{2} / \mathrm{N}_{2}$, $\mathrm{H}_{2} / \mathrm{CH}_{4}$ 的理想分离系数分别为 $9.7,5.1,3.1$ (表 1 ), 均超 过了所对应的努森扩散系数 $\left(\mathrm{H}_{2} / \mathrm{CO}_{2}: 4.7, \mathrm{H}_{2} / \mathrm{N}_{2}: 3.7\right.$, 
$\mathrm{H}_{2} / \mathrm{CH}_{4}: 2.8$ ), 表明 DMTA-COF 具有一定的 $\mathrm{H}_{2}$ 分离和纯 化的潜在应用价值.

表 1 常温常压下, DMTA-COF 膜的单组分气体的分离性能

Table 1 Single gas separation properties of DMTA-COF membrane at room temperature and atmospheric pressure

\begin{tabular}{ccccc}
\hline $\mathrm{Gas}(i / j)$ & $\mathrm{KC}^{a}$ & Permeance $^{b}$ & Permeance & $\mathrm{ISF}^{c}$ \\
\hline $\mathrm{H}_{2} / \mathrm{CO}_{2}$ & 4.7 & & 0.68 & 9.7 \\
$\mathrm{H}_{2} / \mathrm{N}_{2}$ & 3.7 & \multirow{2}{*}{6.6} & 1.3 & 5.1 \\
$\mathrm{H}_{2} / \mathrm{CH}_{4}$ & 2.8 & & 2.1 & 3.1 \\
\hline
\end{tabular}

${ }^{a} \mathrm{KC}$ : Knudsen constant; ${ }^{b}$ Permeance: $\times 10^{-7} \mathrm{~mol} \cdot \mathrm{m}^{-2} \cdot \mathrm{s}^{-1} \cdot \mathrm{Pa}^{-1}$. ${ }^{c}$ ISF: Ideal separation factor.

随后, 对 DMTA-COF 膜在常温常压条件下进行了 等物质的量双组分气体的测试, 如表 2 所示. 混合双组 分气体的分离系数由两种气体在渗透端和残留端的物 质的量分数之比求得, 经过计算 $\mathrm{H}_{2} / \mathrm{CO}_{2}$ 混合双组分气 体的分离系数为 8.3 , 同样超过了所对应的努森扩散系 数(4.7), 也超过了之前文献报道的 COF 膜, 例如 Caro 等 ${ }^{[29]}$ 在 3-氨丙基三乙氧基硅烷(APTES)修饰的多孔氧化 铝基底上制备的 COF- LZU1 膜 $\left(\mathrm{H}_{2} / \mathrm{CO}_{2}:\right.$ 5.99)以及 2016 年由我们课题组 ${ }^{[28]}$ 在聚苯胺(PANI)修饰的多孔二氧化 硅基底上制备的首个可用于气体分离的 COF-300 膜 $\left(\mathrm{H}_{2} / \mathrm{CO}_{2}\right.$ : 6.0). 目前为止, $\mathrm{COF}$ 膜具有的最高的 $\mathrm{H}_{2} / \mathrm{CO}_{2}$ 分离系数仅为 14 , 这主要是由于 $\mathrm{COF}$ 较大的孔尺寸 $(0.8 \sim 5 \mathrm{~nm})$, 弱化了尺寸笁分效应, 从而限制了 $\mathrm{COF}$ 膜 的气体分离选择性. 通过选取含有醚氧基团的 DMTA 作为建构单元制备了 DMTA- COF 膜, 在希弗碱类型 的 $\mathrm{COF}$ 基础上引入了大量的醚氧基团, 大大提高了其 对 $\mathrm{CO}_{2}$ 的亲和力, 增强了 DMTA-COF 膜的化学吸附效 应, 最终得到了较高的 $\mathrm{H}_{2} / \mathrm{CO}_{2}$ 分离选择性能.

表 2 常温常压下, DMTA-COF 膜的 $1: 1$ 的混合双组分气体的分离性 能

Table 2 The mixture gases separation properties of the DMTA-COF membrane at room temperature and atmospheric pressure with 1:1 binary mixtures

\begin{tabular}{ccccc}
\hline Gas $(i / j)$ & $\mathrm{KC}^{a}$ & Permeance $^{b}$ & Permeance & $\mathrm{SF}^{c}$ \\
\hline $\mathrm{H}_{2} / \mathrm{CO}_{2}$ & 4.7 & 6.3 & 0.99 & 8.3 \\
$\mathrm{H}_{2} / \mathrm{N}_{2}$ & 3.7 & 6.4 & 1.7 & 4.5 \\
$\mathrm{H}_{2} / \mathrm{CH}_{4}$ & 2.8 & 8.0 & 4.0 & 2.3 \\
\hline
\end{tabular}

${ }^{a}$ KC: Knudsen constant. ${ }^{b}$ Permeance: $\times 10^{-7} \mathrm{~mol} \cdot \mathrm{m}^{-2} \cdot \mathrm{s}^{-1} \cdot \mathrm{Pa}^{-1} \cdot{ }^{c} \mathrm{SF}$ : Separation factor.

从表 2 中还可以看出, 在 $\mathrm{H}_{2} / \mathrm{CO}_{2}$ 双组分气体测试中 $\mathrm{H}_{2}$ 的渗透通量为 $6.3 \times 10^{-7} \mathrm{~mol} \cdot \mathrm{m}^{-2} \cdot \mathrm{s}^{-1} \cdot \mathrm{Pa}^{-1}$, 比单组分 $\mathrm{H}_{2}$ 渗透通量略低, 这是由于大量吸附在 DMTA-COF 膜 孔道中的 $\mathrm{CO}_{2}$ 在一定程度上阻碍了 $\mathrm{H}_{2}$ 的渗透. 在 $\mathrm{H}_{2} / \mathrm{CO}_{2}$ 双组分气体渗透过程中, 尺寸较小的 $\mathrm{H}_{2}$ 分子可 以快速并且顺利地在孔道中扩散, 进而穿透膜材料, 而 由于 $\mathrm{H}_{2}$ 与 $\mathrm{CO}_{2}$ 分子存在相互竞争作用, 所以大量占据 DMTA-COF 孔道的 $\mathrm{H}_{2}$ 分子阻碍了 $\mathrm{CO}_{2}$ 的渗透, 从而导
致 $\mathrm{CO}_{2}$ 渗透通量降低, 有助于增强 $\mathrm{H}_{2} / \mathrm{CO}_{2}$ 的分离选择 性; DMTA-COF 膜材料对 $\mathrm{CO}_{2}$ 具有较强的选择吸附作 用, 大量 $\mathrm{CO}_{2}$ 分子被吸附在孔道中, 阻碍了其他气体分 子的顺利通过, 其中对动力学直径大的 $\mathrm{CO}_{2}$ 的渗透阻碍 作用较大, 对动力学直径小的 $\mathrm{H}_{2}$ 渗透的影响则较小, 最 终导致 $\mathrm{CO}_{2}$ 渗透通量降低的程度远远大于 $\mathrm{H}_{2}$, 同样有 利于 $\mathrm{H}_{2} / \mathrm{CO}_{2}$ 的分离选择性的增强. 综上所述, DMTA-COF 膜具有较高的 $\mathrm{H}_{2} / \mathrm{CO}_{2}$ 分离性能, 主要归因 于由动力学直径引起的扩散差异以及由选择性吸附导 致的吸附差异的协同作用.

$\mathrm{H}_{2} / \mathrm{N}_{2}$ 双组分气体的分离系数为 4.5 , 略高于所对应 的努森扩散系数 (3.7); $\mathrm{H}_{2} / \mathrm{CH}_{4}$ 的分离系数为 2.3 , 与所对 应的努森扩散系数 (2.8)相差不大, 这与 $\mathrm{Gao}$ 等 ${ }^{[38]}$ 在 APTES 修饰的多孔氧化铝基底上制备的 COF-320 膜所 表现出的 $\mathrm{H}_{2} / \mathrm{N}_{2}$ 和 $\mathrm{H}_{2} / \mathrm{CO}_{2}$ 的分离系数很接近 $\left(\mathrm{H}_{2} / \mathrm{N}_{2}: 3.5\right.$; $\mathrm{H}_{2} / \mathrm{CH}_{4}$ : 2.5). DMTA-COF 具有较低的 $\mathrm{H}_{2} / \mathrm{N}_{2}$ 和 $\mathrm{H}_{2} / \mathrm{CH}_{4}$ 分离系数, 这是由于 DMTA-COF 的孔尺寸较大 $(1.06$ $\mathrm{nm})$, 远远大于气体分子的动力学直径, 在很大程度上 弱化了尺寸篮分效应，导致气体在 DMTA-COF 膜中的 渗透主要由努森扩散主导; 另一方面, 在常温常压下, DMTA-COF 对 $\mathrm{H}_{2}, \mathrm{~N}_{2}$ 和 $\mathrm{CH}_{4}$ 的吸附作用较弱, 这就导致 在 $\mathrm{H}_{2} / \mathrm{CH}_{4}, \mathrm{H}_{2} / \mathrm{N}_{2}$ 气体渗透过程中很难通过吸附差异增 强气体分离选择性能. 这也就是 DMTA-COF 膜的 $\mathrm{H}_{2} /$ $\mathrm{CO}_{2}$ 的分离系数远高于 $\mathrm{H}_{2} / \mathrm{N}_{2}$ 和 $\mathrm{H}_{2} / \mathrm{CH}_{4}$ 的原因.

众所周知, 膜的气体分离选择性能的好坏由气体的 渗透性和选择性共同决定，而这两者又存在相互制衡的 关系，即当膜材料具有较高的气体渗透性的时候，其选 择性往往比较低, 反之亦然. 如果一种膜材料具有较高 的选择性, 而其渗透性比较低, 就会大大降低气体分离 效率; 而如果当膜材料具有非常大的气体渗透性的时候 则会降低甚至失去分离的效果. 所以，如何制备具有高 的选择性的同时又具备较高的渗透性的气体分离膜一 直以来都是研究者的追求. 图 12 是 $\mathrm{H}_{2} / \mathrm{CO}_{2}$ 双组分气体 的选择性和所对应的 $\mathrm{H}_{2}$ 的渗透性的关系图. 从图中可 以看出，与文献报道的 COF 膜相比, DMTA-COF 膜表现 出了高选择性和高渗透性，并且超过了最新的“罗宾逊” 上限 ${ }^{[39]}$, 表明 DMTA-COF 膜具有高的 $\mathrm{H}_{2} / \mathrm{CO}_{2}$ 渗透分离 选择性能.

\section{3 结论}

总之，在反应釜中通过溶剂热的方法制备得到了一 种新型的含有醚氧基团的 3D 共价有机骨架(DMTA$\mathrm{COF}$ ), 由于骨架中含有大量的醚氧基团，该材料对 $\mathrm{CO}_{2}$ 表现出了高的亲和力和捕获力. 基于 DMTA-COF 较强 的 $\mathrm{CO}_{2}$ 选择吸附作用, 在聚苯胺修饰的多孔氧化铝基底 上利用共价功能化连接通过原位合成的方法制备得到 了致密连续的 DMTA-COF 膜. 通过分子尺寸篮分效应 和吸附效应的协同作用, DMTA-COF 膜表现出了高的 


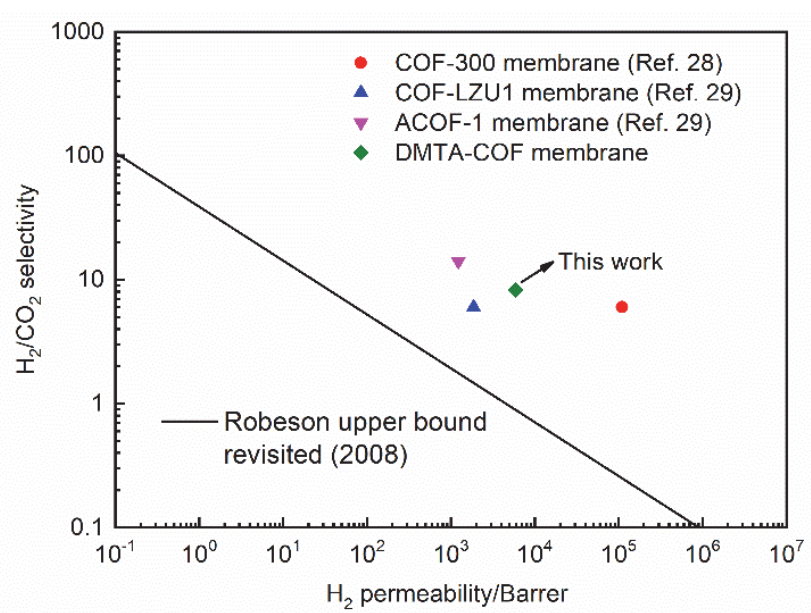

图 12 DMTA-COF 膜和文献报道的 COF 膜的 $\mathrm{H}_{2} / \mathrm{CO}_{2}$ 混合气体的选 择性和 $\mathrm{H}_{2}$ 渗透性与 Robeson upper bound (2008)的关系

Figure $12 \quad \mathrm{H}_{2} / \mathrm{CO}_{2}$ selectivity and $\mathrm{H}_{2}$ permeability of DMTA-COF membrane and other reported COF membrane in relation to Robeson's upper bound (2008)

Permeability is calculated as the membrane permeance multiplied by the membrane thickness $\left(\mathrm{mol} \cdot \mathrm{m}^{\circ} \mathrm{m}^{-2} \cdot \mathrm{s}^{-1} \cdot \mathrm{Pa}^{-1}\right), 1$ Barrer $=3.348 \times 10^{-16}$ $\mathrm{mol} \cdot \mathrm{m} \cdot \mathrm{m}^{-2} \cdot \mathrm{s}^{-1} \cdot \mathrm{Pa}^{-1}$

$\mathrm{H}_{2}$ 渗透选择性, 其中 $\mathrm{H}_{2} / \mathrm{CO}_{2}$ 的分离系数为 $8.3, \mathrm{H}_{2}$ 的渗 透通量高达 $6.3 \times 10^{-7} \mathrm{~mol} \cdot \mathrm{m}^{-2} \cdot \mathrm{s}^{-1} \cdot \mathrm{Pa}^{-1}$, 表明 DMTA$\mathrm{COF}$ 膜在 $\mathrm{H}_{2}$ 的纯化和分离领域具有较大的应用前景.

\section{4 实验部分}

\section{1 试剂与仪器}

聚苯胺 $\left(M_{\mathrm{w}}=1.5 \times 10^{4}\right)$ 购买于西格玛奥德利奇 $($ 中 国); 四胺基四苯甲烷( $98 \%$ )购买于吉林中科研伸科技有 限公司; 2,5-二甲氧基对苯二甲醛(97\%)购买于安耐吉化 学技术 (上海) 有限公司; 邻二氯苯 $(99 \%)$, 正丁醇 (99.8\%), 二氧六环 $(99 \%)$ 和氢化钲( $95 \%)$ 均购买于上海 阿拉丁生化科技股份有限公司; 冰醋酸 $(99.5 \%)$, 乙醇 $(\geqslant 99.5 \%)$ 和四氢呋喃 $(99.5 \%)$ 购买于西陇化工股份有限 公司. 邻二氯苯, 正丁醇, 二氧六环以及四氢呋喃使用 前通过氢化钙蒸馏进行除水.

日本岛津 IRAFFINITY-1 型傅里叶变换红外光谱仪; SHIMADZU DTG-60 的差热热重热分析系统; 日本 SHIMADZU XRD-6000 X-Ray粉末 X 射线衍射仪; 日本 JEOS JSM 6700 扫描电子显微镜; 美国麦克公司的 Micro Meritics Tristar II 3020 型气体吸附测试仪器.

\section{2 实验合成过程}

\subsection{1 $\mathrm{Al}_{2} \mathrm{O}_{3}$ 基底的预处理和修饰}

$\mathrm{Al}_{2} \mathrm{O}_{3}$ 基底的预处理和利用聚苯胺对其进行修饰的 方法参考文献报道 ${ }^{[25]}$.

在对氧化铝基底进行聚苯胺修饰后, 接着对其进行 了醛基化修饰. 在电子天平上准确称量 $20.00 \mathrm{mg} 2,5$-二 甲氧基对苯二甲醛于 $25.00 \mathrm{~mL}$ 的反应釜中; 随后用量
程为 $5 \mathrm{~mL}$ 的注射器量取 $5 \mathrm{~mL}$ 无水的二氧六环溶剂于上 述反应釜中，超声 $5 \mathrm{~min}$ ，使 2,5-二甲氧基对苯二甲醛完 全溶解, 得到澄清透明的黄色溶液; 接着把聚苯胺修饰 的多孔氧化铝基底置于上述反应釜中, 并且使修饰聚苯 胺的一面朝上放置; 装好反应釜, 于 $150{ }^{\circ} \mathrm{C}$ 烘箱中反应 $1 \mathrm{~h}$. 反应结束后, 取出反应釜冷却至室温, 用大量无水 的二氧六环反复洗涤基底表面, 置于无水四氢呋喃溶剂 中过夜, 最后于室温下干燥, 备用.

\subsubsection{DMTA-COF 的制备}

在电子天平上准确称量 $25.60 \mathrm{mg} 2,5$-二甲氧基对苯 二甲醛(DMTA)和 $25.20 \mathrm{mg}$ 四胺基四苯甲烷(TAM)于 $25.00 \mathrm{~mL}$ 反应釜中, 随后用量程为 $2.00 \mathrm{~mL}$ 的注射器分 别量取 $1.50 \mathrm{~mL}$ 无水邻二氯苯和 $1.50 \mathrm{~mL}$ 无水正丁醇于 上述反应釜中, 并且超声 $5 \mathrm{~min}$ 使单体分散完全, 得到 黄色的乳浊液. 接着在摚拌下, 向得到的乳浊液中缓慢 滴加 $0.30 \mathrm{~mL}$ 浓度为 $6.00 \mathrm{~mol} \cdot \mathrm{L}^{-1}$ 的醋酸水溶液. 最后 迅速装好反应釜于 $100{ }^{\circ} \mathrm{C}$ 反应 $3 \mathrm{~d}$. 反应结束后, 冷却 至室温, 通过过滤得到黄色沉淀, 并且用无水四氢呋喃 反复洗涤. 产物浸泡于无水四氢呋喃中过夜, 再次过滤, 产物于 $100{ }^{\circ} \mathrm{C}$ 真空干燥 $12 \mathrm{~h}$, 最终得到 $38.10 \mathrm{mg}$ 黄色的 DMTA-COF 粉末, 产率为 75\%.

\subsubsection{DMTA-COF 膜的制备}

分别准确称量 2,5-二甲氧基对苯二甲醛 $(25.60 \mathrm{mg}$ ) 和四胺基四苯甲烷 $(25.20 \mathrm{mg}$ )于 $25.00 \mathrm{~mL}$ 反应釜中，随 后用量程为 $2.00 \mathrm{~mL}$ 的注射器依次量取无水正丁醇 $(1.50$ $\mathrm{mL})$ 和无水邻二氯苯 $(1.50 \mathrm{~mL})$ 于上述反应釜中. 接着超 声处理 $5 \mathrm{~min}$, 使单体在溶剂中混合均匀, 分散完全, 得 到黄色的乳浊液. 量取醋酸水溶液 $\left(6 \mathrm{~mol} \cdot \mathrm{L}^{-1}, 0.30 \mathrm{~mL}\right)$ 在摚拌下缓慢滴加到上述乳浊液中, 滴加完毕后把修饰 好的基底使得修饰层朝上水平放入反应釜中，迅速装好 反应釜，于 $100{ }^{\circ} \mathrm{C}$ 反应 $3 \mathrm{~d}$. 反应结束后，冷却至室温， 取出 DMTA-COF 膜, 用无水四氢呋喃反复冲洗膜表面, 接着把膜置于无水四氢呋喃中过夜, 最终把 DMTACOF 膜于 $60{ }^{\circ} \mathrm{C}$ 抽真空处理 $10 \mathrm{~h}$.

\section{3 气体分离测试}

气体的峰面积是通过日本 SHIMADZU GC-14C 气 相色谱进行测试的, 以 $\mathrm{TCD}$ 为检测器, 载气为 $\mathrm{He}$, 检测 器温度设置为 $120{ }^{\circ} \mathrm{C}$, 柱箱温度设置为 $30{ }^{\circ} \mathrm{C}$. 气体分 离装置为自制的, 与气相色谱连用. 所有气体的渗透测 试均是在常温和常压下进行. 单组分气体测试过程中, $\mathrm{Ar}$ 为吹扫气, $\mathrm{H}_{2}, \mathrm{CO}_{2}, \mathrm{~N}_{2}, \mathrm{CH}_{4}$ 为原料气. 载气流速为 30 $\mathrm{mL} \cdot \mathrm{min}^{-1}$, 原料气流速为 $30 \mathrm{~mL} \cdot \mathrm{min}^{-1}$. 单组分气体的 理想分离系数 $\alpha$.

$$
\alpha_{i / j}=\frac{P_{i}}{P_{j}}
$$

其中 $\alpha_{i j}$ 代表气体 $i / j$ 的理想分离系数; $i, j$ 分别代表气体 $i$ 和气体 $j ; P$ 代表气体的渗透通量 $\left(\mathrm{mol} \cdot \mathrm{m}^{-2} \cdot \mathrm{s}^{-1} \cdot \mathrm{Pa}^{-1}\right)$.

双组分气体的分离测试过程中, 以 $\mathrm{Ar}$ 为吹扫气, 
$\mathrm{H}_{2} / \mathrm{CO}_{2}, \mathrm{H}_{2} / \mathrm{N}_{2}, \mathrm{H}_{2} / \mathrm{CH}_{4}$ 为原料气. 吹扫气流速为 30 $\mathrm{mL} \cdot \mathrm{min}^{-1}$, 原料气为 $1: 1$ 的两种气体的混合气体, 每 种气体流量均为 $30 \mathrm{~mL} \cdot \mathrm{min}^{-1}$, 测试之前, 各路气体进 行配气需要稳定 $3 \mathrm{~h}$. 双组分气体的分离系数 $\alpha$ :

$$
\alpha_{i / j}=\frac{x_{i} / x_{j}}{y_{i} / y_{j}}
$$

其中 $x_{i}, x_{j}$ 分别为渗透端气体 $i$ 和气体 $j$ 的摩尔分数; $y_{i}, y_{j}$ 分别为残留端气体 $i$ 和 $j$ 的摩尔分数.

\section{References}

[1] Koros, W. J.; Zhang, C. Nat. Mater. 2017, 16, 289.

[2] Huang, A.-S.; Liang, F.-Y.; Steinbach, F.; Caro. J. J. Membr. Sci. 2010, 350, 5 .

[3] Huang, A.-S.; Caro, J. J. Mater. Chem. 2011, 21, 11424.

[4] Shi, K.-Y.; Chi, Y.-J.; Jin, X.-Q.; Xu, M.; Yuan, F.-L.; Fu, H.-G. Acta Chim. Sinica 2005, 63, 885. (史克英, 池玉娟, 金效齐, 徐敏, 袁 福龙, 付宏刚, 化学学报, 2005, 63, 885.)

[5] Huang, A.-S.; Wang, N.-Y.; Kong, C.-L.; Caro, J. Angew. Chem. Int. Ed. 2012, 51, 10551 .

[6] Ben, T.; Lu, C.-J.; Pei, C.-Y.; Xu, S.-X.; Qiu, S.-L. Chem. Eur. J. 2012, 18, 10250

[7] Liu, B.; Tang, L.-X.; Lian, Y.-H.; Li, Z.; Sun, C.-Y.; Chen, G.-J. Acta Chim. Sinica 2013, 71, 920. (刘蓓, 唐李兴, 廉源会, 李智, 孙长 宇, 陈光进, 化学学报, 2013, 71, 920.)

[8] Guo, H.-L.; Zhu, G.-S.; Hewitt, I. J.; Qiu, S.-L. J. Am. Chem. Soc. 2009, 131, 1646

[9] Budd, P. M.; Msayib, K. J.; Tattershall, C. E.; Ghanem, B. S.; Reynolds, K. J.; McKeown, N. B.; Fritsch, D. J. Membr. Sci. 2005, $251,263$.

[10] Diercks, C. S.; Yaghi, O. M. Science 2017, 355, 6328.

[11] Chen, Q.-D.; Tang, J.-J.; Fang, Q.-R. Chem. J. Chin. Univ. 2018, 39, 2357. (陈奇丹, 唐俊杰, 方千荣, 高等学校化学学报, 2018, 39, 2357.)

[12] Huang, N.; Wang, P.; Jiang, D.-L. Nat. Rev. Mater. 2016, 1, 16068.

[13] Wang, Z.-T.; Li, H.; Yan, S.-C.; Fang, Q.-R. Acta Chim. Sinica 2020, 78, 63. (王志涛, 李辉, 颜士臣, 方千荣, 化学学报, 2020, 78, 63.)

[14] Huang, W.; Li, Y.-G. Chin. J. Chem. 2019, 37, 1291

[15] Peng, Z.-K.; Ding, H.-M.; Chen, R.-F.; Gao, C.; Wang, C. Acta Chim. Sinica 2019, 77, 681. (彭正康, 丁慧敏, 陈如凡, 高超, 汪 成, 化学学报, 2019, 77, 681.)
[16] Dong, G.-X.; Lee, Y. M. J. Mater. Chem. A 2017, 5, 13294.

[17] Yuan, S.-S.; Li, X.; Zhu, J.-Y.; Zhang, G.; Puyvelde, P. V.; Bruggen, B. V. Chem. Soc. Rev. 2019, 48, 2665

[18] Wang, J.; Zhu, J.-Y.; Zhang, Y.-T.; Liu, J.-D.; Bruggen, B. V. Nanoscale 2017, 9, 2942.

[19] Ding, S.-Y.; Wang, W. Chem. Soc. Rev. 2013, 42, 548

[20] Uribe-Romo, F. J.; Doonan, C. J.; Furukawa, H.; Oisaki, K.; Yaghi, O. M. J. Am. Chem. Soc. 2011, 133, 11478.

[21] Kandambeth, Sharath.; Mallick, A.; Lukose, B.; Mane, M. V.; Heine, T.; Rahul, B. J. Am. Chem. Soc. 2012, 134, 19524.

[22] Zhou, H.-C.; Long, J.-R.; Yaghi, O. M. Chem. Rev. 2012, 112, 673.

[23] Chung, T. S.; Jiang, L.-Y.; Li, Y.; Kulprathipanja, S. Prog. Polym. Sci. 2007, 32, 483.

[24] Bunck, D. N.; Dichtel, W. R. J. Am. Chem. Soc. 2013, 135, 14952.

[25] Liu, X.-H.; Guan, C.-Z.; Ding, S.-Y.; Wang, W.; Yan, H.-Y.; Wang, D.; Wan, L.-J. J. Am. Chem. Soc. 2013, 135, 28, 10470.

[26] Dai, W.-Y.; Shao, F.; Szczerbiński, J.; McCaffrey, R.; Zenobi, R.; Jin, Y.-H.; Schlüter, D.; Zhang, W. Angew. Chem., Int. Ed. 2016, 55, 213.

[27] Dey, K.; Pal, M.; Rout, K. C.; Kunjattu-H, S.; Das, A.; Mukherjee, R.; Kharul, U. K.; Baneriee, R. J. Am. Chem. Soc. 2017, 139, 13083.

[28] Fu, J.-R.; Das, S.; Xing, G.-L.; Ben, T.; Valtchev, V.; Qiu, S.-L. J. Am. Chem. Soc. 2016, 138, 7673 .

[29] Fan, H.-W.; Mundstock, A.; Feldhoff, A.; Knebel, A.; Gu, J.-H.; Meng, H.; Caro, J. J. Am. Chem. Soc. 2018, 140, 10094.

[30] Fan, H.-W.; Mundstock, A.; Gu, J.-H.; Meng, H.; Caro, J. J. Mater. Chem. A. 2018, 6, 16849 .

[31] Segura, J. L.; Mancheo, M. J.; Zamora, F. Chem. Soc. Rev. 2016, 45, 5635.

[32] Ma, Y.-X.; Li, Z.-J.; Wei, L.; Ding, S.-Y.; Zhang, Y.-B.; Wang, W. J. Am. Chem. Soc. 2017, 139, 4995.

[33] Zhang, Y.-B.; Su, J.; Furukawa, H.; Yun, Y.-F.; Gándara, F.; Duong, A.; Zou, X.-D.; Yaghi, O. M. J. Am. Chem. Soc. 2013, 135, 16336.

[34] Bureekaew, S.; Sato, H.; Matsuda, R.; Kubota, Y.; Hirose, R.; Kim, J.; Kato, K.; Takata, M.; Kitagawa, S. Angew. Chem., Int. Ed. 2010, 49,7660 .

[35] Reichenbach, C.; Kalies, G.; Lincke, J.; Lässig, D,; Krautscheid, H.; Moellmer, J.; Thommes, M. Microporous Mesoporous Mater. 2011, $142,592$.

[36] Feng, S.-C.; Ren, J.-Z.; Li, H.; Hua, K.-S.; Li, X.-X.; Deng, M.-C. Membr. Sci. Technol. 2013, 33, 53. (冯世超, 任吉中, 李晖, 花开 胜, 李新学, 邓麦村, 膜科学与技术, 2013, 33, 53)

[37] Lin, H.-Q.; Freeman, B. D. J. Mol. Struct. 2005, 739, 57.

[38] Lu, H.; Wang, C.; Chen, J.-J.; Ge, R.-L.; Leng, W.-G.; Dong, B.; Huang, J.; Gao, Y.-N. Chem. Commun. 2015, 51, 15562.

[39] Robeson, L. M. J. Membr. Sci. 2008, 320, 390. 